\title{
Image Segmentation Using the EM/MPM Algorithm
}

\author{
Hanane DALIMI*, Mohamed AFIFI, Said AMAR \\ Engineering and Materials Laboratory, Engineering and Energy Team, Ben M'sik Faculty of Sciences, \\ Casablanca, Morocco \\ Corresponding Author : * hanane.dalimi@gmail.com
}

\section{ABSTRACT}

\section{Article Info}

Volume 8, Issue 3

Page Number : 618-622

\section{Publication Issue}

May-June-2021

Article History

Accepted : 01 June 2021

Published : 15 June 2021
In this article we propose to place our work in a Markovian framework for unsupervised image segmentation. We give one of the procedures for estimating the parameters of a Markov field, we limit the work to the EM estimation method and the Posterior Marginal Maximization (MPM) segmentation method. Estimating the number of regions who compones the image is relatively difficult, we try to solve this problem by the $\mathrm{K}$-means Histogram method.

Keywords: Unsupervised Segmentation, EM algorithm, K-means, Histogram Markov, MPM

\section{INTRODUCTION}

The image segmentation is the division of an image into regions or categories which correspond to different objects or parts of objects. Every pixel in an image is allocated to one of a number of these categories.

Many segmentation approaches can be found in the literature generally classified into three basic groups [1], segmentation based on edges and segmentation according to regions. Recently several other categories have been considered such as color-based techniques [2], neural networks and fuzzy logic [3] and genetic algorithms [4] \& [5]. In the case of unsupervised segmentation, the statistical properties of the classes are unknown and must be estimated.
The EM/MPM algorithm is a stochastic algorithm which this paper addresses the problem of segmenting images. The method we used EM/MPM combines the expectation-maximization (EM) algorithm for parameter estimation with the MPM algorithm for segmentation and the algorithm initialize by the $\mathrm{K}$ means Histogram method. We describe the method in Sections II and present the experimental results in Section III, followed by our conclusions.

\section{MARKOVIAN RANDOM FIELD MODEL TO SEGMENTATION}

Markov fields have been used for twenty years now in image processing and are now part of the basic techniques of this discipline. Originally born in the context of statistical physics to study phase transition 
phenomenon, they are quickly applied to twodimensional networks that make up images. The first models are restricted by causal constraints and therefore limited until the founding article by Geman in 1982 [6], which really opens up their use in image processing. This use will increase over the years and will give rise to a great deal of research into the problems that their use gives rise to calculation of model parameters, simulation techniques and the search for quick solutions, etc.. Alongside this still topical work, new research has begun dedicated to higher-level tasks and aimed at the interpretation of images. The use of Markovian techniques in image processing remains today a fundamental point in the field of segmentation, restoration or even stochastic modeling.

Markov fields are used to extract local contextual information from an image. For these models, the intensity of each pixel depends only on the gray level of the neighboring pixels. They define a very efficient method for defining nonlinear interactions between parameters of the same or different nature, such as gray level and spatial position. They have been applied to image segmentation.

\section{A. The EM/MPM segmentation}

The stochastic method of unsupervised EM/MPM image segmentation was originally proposed by Comer and Delp [7], the observed data is the observed image, given by $Y=Y_{1}, \ldots, Y_{N}$ and the label field $X=X_{1}, \ldots, X_{N}$ is the missing data, $X$ and $Y$ are 2D random fields of a rectangular grid $S$ with $N$ variables. According to Bayes Theorem, the posterior probability $P_{X \mid Y}$ is proportional to the prior probability $p_{X}$ times a likelihood function $f_{Y \mid X}$. Hence:

$$
P_{Y \mid X}(Y \mid X, \theta)=p_{X}(X) * f_{Y \mid X}(Y \mid X, \theta)
$$

where $\theta$ is the parameter array for the model , $y=$ $y_{1}, \ldots, y_{n}$ and $x=x_{1}, \ldots, x_{n}$ are sample realizations of
$Y$ and $X$ respectively. Each $x_{s}$ can be assigned the value of a label $k=1, \ldots, L$ with $L$ being the number of labels previously defined. On the other hand, $y_{s}$ are grey levels in the range $0, \ldots, 255$.

The segmentation methods seeks to find an estimate for the label field $\mathrm{x}$ through the MPM optimization method, whose minimization criterion is the expected number of mislabelled pixels. As an unsupervised segmentation method, the EM algorithm is then employed to estimate the parameters $\theta$ of the model.

In the original formulation proposed by Comer and Delp [7] for image segmentation, the probabilit $f_{Y_{S} \mid X} \forall s \in\{1, \ldots, N\}$ is considered a normal distribution that only depends on $X_{S}$.

Hence:

$$
\begin{aligned}
& f_{Y \mid X}(Y \mid X, \theta)=\prod_{s=1}^{N} f_{Y_{S} \mid X, \theta}\left(y_{s} \mid X, \theta\right) \\
& =\prod_{s=1}^{N} \frac{1}{\sqrt{2 \pi \sigma_{x_{s}}^{2}}} \exp \left(-\frac{\left(y_{s}-\mu_{x_{s}}\right)^{2}}{2 \sigma_{x_{s}}^{2}}\right)
\end{aligned}
$$

The array of parameters $\theta$ are the means and variance of each label, ie. $\theta=\left[\mu_{1}, \sigma_{2}, \ldots, \mu_{L}, \sigma_{2 L}\right]$.

The $p_{X}$ distribution of the label fiels is a Markovian random field which is according to the HammersleyClifford theorem a mass function of the conditional conversation given as a Gibbs distribution.

$$
P(X=x)=\frac{1}{Z} \exp (-U(x))
$$

where $Z$ is a normalisation factor and $U(x)$ is an energy function in the form:

$$
U(x)=\sum_{\{r, s\} \in C} \beta t\left(X_{r}, X_{S}\right)+\sum_{\{r\} \in C} \gamma_{X_{r}}
$$

$\beta=$ weighting factor for amount of spatial interaction

$\gamma_{X_{r}}=$ cost factor for class $\mathrm{k}$, used for ultrasound attenuation. 
The EM algorithm has been successfully used for estimating Gauss parameters $\theta$ during the EM/MPM segmentation. EM iterates through two steps. First the expectation step creates the current estimates of the hyper-parameters $\theta$, then the maximization step.

If $\theta^{p}$ is the estimation of $\theta$ at the $p$ th iteration, then the expectation function at $p$ th iteration is:

$$
\begin{aligned}
Q\left(\theta, \theta_{p-1}\right)=E & {\left[\log f(y \mid x, \theta) \mid Y, \theta_{p-1}\right] } \\
& +E\left[\log p(x \mid \theta) \mid Y, \theta_{p-1}\right]
\end{aligned}
$$

\section{B. Initialization of classes}

The class initiation step consists of a preprocessing aimed at initializing the parameters of the EM algorithm and ensuring better identification of the mixture. In this context, several methods have been developed, the most popular are those of K-means and Histogram.

However, the main limitation of the K-means method is also the dependence of the results on the starting values (initial centers). Each initialization corresponds to a different solution (local optimum) which can in certain cases be very far from the optimum solution (global optimum).

In this part two techniques are applied:

The first one is to calculate the histogram of the image, detect the histogram peaks and provide their corresponding gray levels;

The second consists in carrying out a presegmentation using the K-means method and the results of the first method.

\section{Segmentation Algorithm Process}

Phase 1: given an original image, calculate his histogram and initialize the mean and the deviation, using the K-means algorithm;

Phase 2: using the EM algorithm, we estimate $\theta$, when the final estimate $\theta$ of the parameters is obtained;
Phase 3: Ordinary segmentation is carried out (supervised segmentation) with known parameters using the MPM algorithm.

\section{RESULTS AND DISCUSSION}

In order, to examine the effectiveness of the method described above, we present his practical side in this part. As in many image segmentation problems, validating the results obtained is a delicate problem. To do this, we first used computer-generated images whose segmentations we know in order to assess the performance of our system. Then, we applied this technique to real images.

In order to assess the performance of our algorithm, we compared it to three other classical segmentation methods, the tests are carried out under Matlab.

Our first experiment is carried out on the synthetic image SAVOISE from the GDR 134 bank. SAVOISE is made up of 6 regions of different shapes and colors.

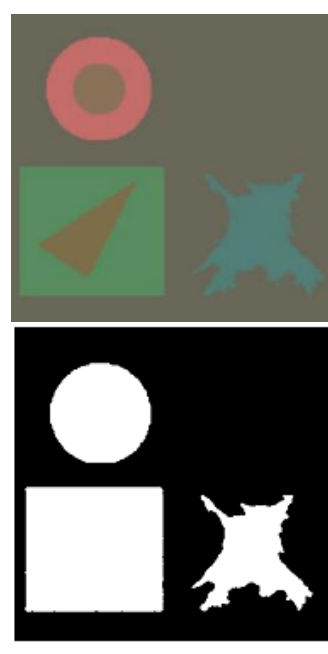

$\mathrm{K}=2$

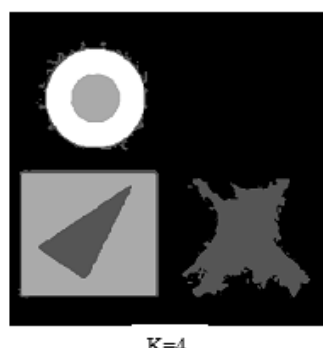

$\mathrm{K}=4$

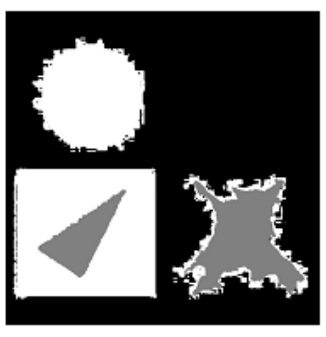

$\mathrm{K}=3$

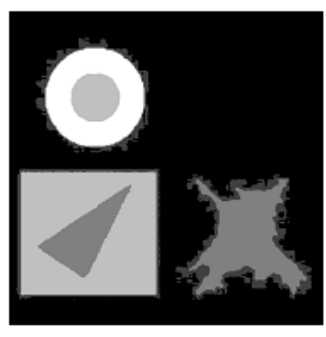

$\mathrm{K}=5$
Figure 1: Savoie image Segmentation result 
The correct classification rate calculated using the PRI index for this result is $88 \%$. The visual assessment is equally satisfactory as it corresponds region by region to the original image. The image has been tested with several values of $K$, the best result is obtained with $\mathrm{K}=4$.

The second experiment is performed on a variety of real-world test images taken from the Berkeley base.

This database contains 300 real world images of $481 \times$ 321 or $321 \times 481$ size, available in color or grayscale.

a

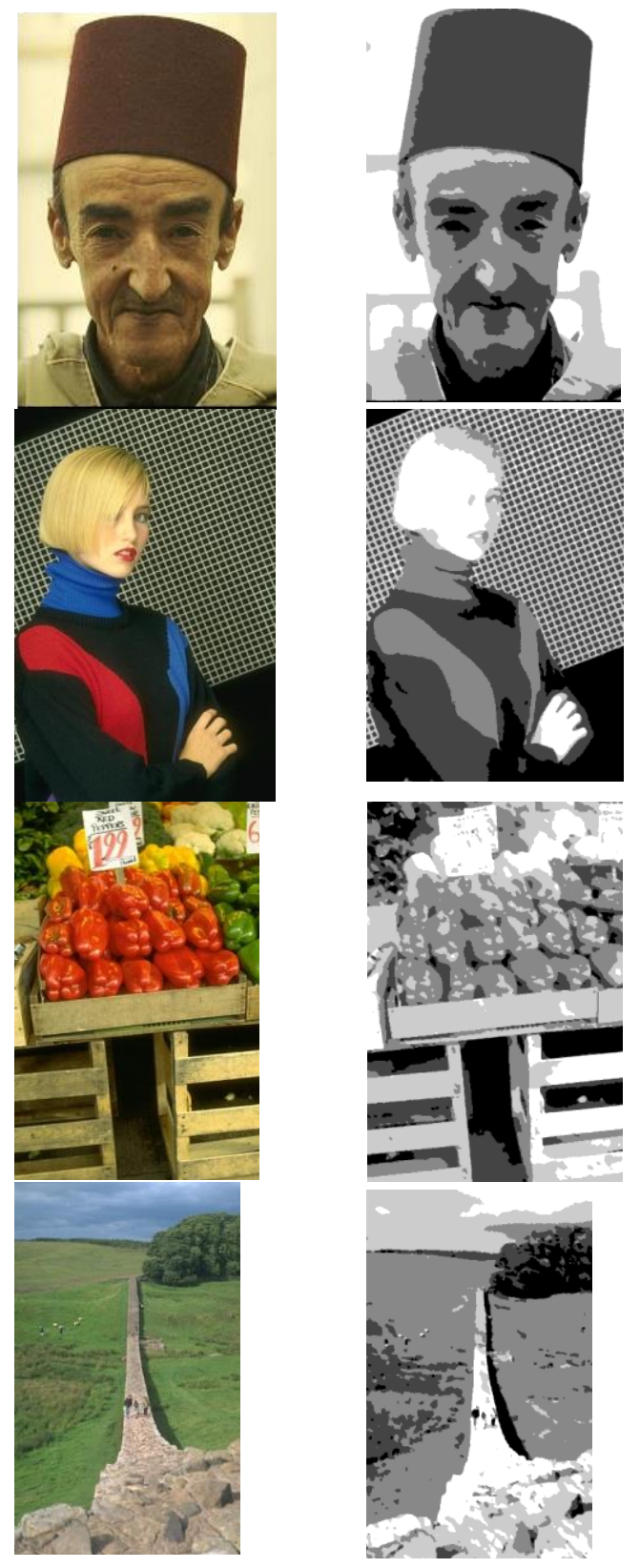

Figure 2 : Segmentation results on images from the Berkeley database
The numerical evaluation of these results obtained using the PRI index is noted in the following table:

Table 1: Performance measures

\begin{tabular}{|l|l|l|l|}
\cline { 2 - 4 } \multicolumn{1}{c|}{} & $\begin{array}{l}\text { Estimated } \\
\text { class number }\end{array}$ & $\begin{array}{l}\text { PRI } \\
(\%)\end{array}$ & $\begin{array}{l}\text { Execution time } \\
\text { (in seconds) }\end{array}$ \\
\hline Image a & 4 & 0.77 & 1.689 \\
\hline Image b & 5 & 0.68 & 1.452 \\
\hline Image c & 5 & 0.88 & 1.352 \\
\hline Image d & 3 & 0.83 & 1.475 \\
\hline
\end{tabular}

The quantitative evaluation reinforces the perceptual quality of the results. The comparison of the results obtained with the semantic segmentation carried out by the experts shows interesting scores that can go up to $88 \%$ of good classification rate. The estimated number of classes also remains reasonable. On the other hand, we notice that the lowest score was registered by images 2, which is equal to $68 \%$ good classification rate. This is due to the small variation in hue between regions.

\section{IV.CONCLUSION}

This article describes a probabilistic approach for unsupervised image segmentation. It differs from other approaches by using the Kmeans-Histogram method proposed in the previous chapter to quickly produce a first segmentation which serves as an initialization for a statistical model MRF (Markov Random Field).

The performance evaluation of the proposed method is established by comparing the segmentation of synthetic images as well as real images with the semantic segmentation carried out by the experts, which makes it possible to establish a visual evaluation. But we also performed quantitative measurements of two commonly used segmentation assessment criteria the PRI.

The experimental results have shown that the quality of the segmentation carried out by the proposed multi-thresholding method is satisfactory. 


\section{REFERENCES}

[1]. R. C. Gonzalez and R. E. Woods, Digital image processing (2nd edition), 1987.

[2]. M. Gonzalez-Rodriguez, D. Benitez-Diaz, and C. P. Suarez-Araujo, "Segmentation and recognition in visual chromatic spaces", Cybernetics and Systems, vol. 21, no. 2-3. pp. 241-247, 1990.

[3]. J. C. Bezdek, "Fuzzy Models for Pattern Recognition: Methods That Search for Structures in Data (Ieee Press Selected Reprint Series).", p. 544, 1992.

[4]. J. Albert, F. Ferri, J. Domingo, and M. Vicens, "an Approach To Natural Scene Segmentation By Means of Genetic Algorithms With Fuzzy Data.", pp. 97-112, 1992.

[5]. C. A. Ankenbrandt, B. P. Buckles, F. E. Petry, and M. Lybanon, "Ocean feature recognition using genetic algorithms with fuzzy fitness functions (GA/F3)", Evolutionary Computation: The Fossil Record. pp. 568-574, 1998.

[6]. S. Geman and D. Geman, Stuart Geman and Donald Geman, "Stochastic Relaxation, Gibbs Distributions, and the Bayesian Restoration of Images", no. 6. Paris, 1982.

[7]. M. L. Comer, E. J. Delp, and W. Lafayette, "the EM/MPM algorithm for segmentation of textured images: analysis and further experimental results", Analysis, 1995.

\section{Cite this article as :}

Hanane DALIMI, Mohamed AFIFI, Said AMAR, "Image Segmentation Using the EM/MPM Algorithm", International Journal of Scientific Research in Science and Technology (IJSRST), Online ISSN : 2395-602X, Print ISSN : 2395-6011, Volume 8 Issue 3, pp. 618-622, May-June 2021. Available at doi : https://doi.org/10.32628/IJSRST218380 Journal URL : https://ijsrst.com/IJSRST218380 